\title{
PAPER
}

\section{Cyclooxygenase inhibition: between the devil and the deep blue sea}

\section{J Hawkey}

Gut 2002;50(Suppl III):iii25-iii30

Non-steroidal anti-inflammatory drugs (NSAIDs) account for more reports of drug related toxicity than any other class of drugs. Their most widely recognised adverse effects are on the gastrointestinal tract. They cause acute erosions and chronic ulcers that result in hospitalisation and death because of ulcer bleeding and perforation. Between them, aspirin and non-aspirin NSAIDs may account for more than half of all episodes of ulcer bleeding and perforation.
Correspondence to: Prof. C J Hawkey, Professor of Gastroenterology, Queen's Medical Centre, University Hospital Nottingham NG7 2UH, UK; cj.hawkey@ nottingham.ac.uk
A recent study has calculated that approximately 2000 patients per annum may die as a result of non-steroidal anti-inflammatory drug (NSAID) induced ulcer bleeding and perforation in the UK. ${ }^{1}$ Important risk factors that have emerged are old age and a past ulcer history, ${ }^{23}$ and these may have additive effects. ${ }^{4}$ NSAID ulcer complications are dose dependent ${ }^{56}$ and the risk is enhanced if patients also take anticoagulants ${ }^{78}$ or corticosteroids, ${ }^{7}$ although whether steroids are an independent risk factor or an NSAID specific risk magnifier remains uncertain. ${ }^{9}$

In addition to these well recognised side effects, NSAIDs affect the entire gastrointestinal tract. ${ }^{2}{ }^{10}$ They are responsible for a high level of dyspepsia, ${ }^{11}$ and cause mucosal damage throughout the gastrointestinal tract. Outside the gastrointestinal tract, NSAIDs can cause fluid retention, ${ }^{12-17}$ hypertension, ${ }^{18}$ and renal impairment. Whether they predispose to or protect against vascular disease is uncertain and recent studies do not establish a clear effect ${ }^{19}$ (Garcia Rodriguez; personal communication ${ }^{20}$ ).

Inhibition of prostaglandin synthesis is well recognised as the central mechanism by which gastrointestinal injury occurs. ${ }^{21}$ This is a result of inhibition of the cyclooxygenase enzyme which converts unsaturated fatty acids such as arachidonic acid (which are released by cell injury) to prostaglandins. In the stomach prostaglandin synthesis is protective as a result of enhanced mucosal blood flow and stimulation of mucus and bicarbonate secretion. ${ }^{22}$ By contrast, in arthritis, prostaglandins mediate pain and some components of inflammation. Recognition that there were two isoforms of cyclooxygenase, with COX-1 predominating in the stomach and an inducible COX-23-25 expressed at sites of inflammation, offered the prospect of separating the beneficial effects of inhibiting prostaglandin synthesis in joints from the harmful effects of inhibiting it in the stomach. Animal studies confirmed that selective inhibitors of COX-2 did not reduce gastric prostaglandin syn- thesis or cause the injury associated with nonselective NSAIDs. Interestingly, a recent study has reported that selective COX-1 inhibitors apparently do not injure the stomach either. ${ }^{26}$ Whether it is generally true that dual inhibition is necessary for gastric injury, and if so how and why this occurs, is however currently not known. Suggestions have also been made for the existence of a third cyclooxygenase enzyme. ${ }^{27}$ A third gene has not emerged from analysis of the human genome but circumstances have been defined in which the drug sensitivity of COX-2 is altered at a post translational level ${ }^{28}$ and where COX-2 may hasten the resolution of inflammation. ${ }^{27}$

\section{AVAILABLE DRUGS: HUMAN EVIDENCE FOR SELECTIVITY}

Selectivity can be shown in both isolated enzyme and whole cell systems. ${ }^{29-31}$ The former tend to result in high estimates of selectivity. A consensus has emerged favouring the use of whole cell systems, specifically the whole blood assay developed by Patrignani and colleagues ${ }^{32}$ (fig 1 ) and recently modified from the William Harvey Institute. ${ }^{31}$ COX- 1 activity is measured as thromoboxane production from platelets in whole blood during clotting under standardised conditions. COX-2 activity is measured (usually over 24 hours) in whole blood stimulated by lipopolysaccharide. COX-2 is induced in monocytes and $\mathrm{PGE}_{2}$ synthesis reflects this. Whole blood assays result in lower estimates of selectivity that are believed more accurately to reflect drug behaviour in vivo.

\section{ROFECOXIB}

Rofecoxib is the most selective of the generation of COX-2 inhibitors that has emerged in the past few years. In the William Harvey modified whole blood assay, it achieved a selectivity ratio of approximately 70 (fig 2). Rofecoxib has been shown in humans to spare gastric prostaglandin synthesis at supratherapeutic doses, in contrast to naproxen $\mathrm{l} \mathrm{g}$ which inhibits gastric mucosal prostaglandin synthesis by about 70\% (fig 3). ${ }^{33} 34$

\section{Acute gastrointestinal (GI) injury}

A remarkable early study showed that rofecoxib $250 \mathrm{mg}$ (10 times the maximum therapeutic dose) given to volunteers for seven days was associated with erosions in $12 \%$, compared to $8 \%$ with placebo, $85 \%$ with ibuprofen $2.4 \mathrm{~g}$ daily, and

Abbreviations: COX, cyclooxygenase; GI, gastrointestinal; NSAID, non-steroidal anti-inflammatory drug 


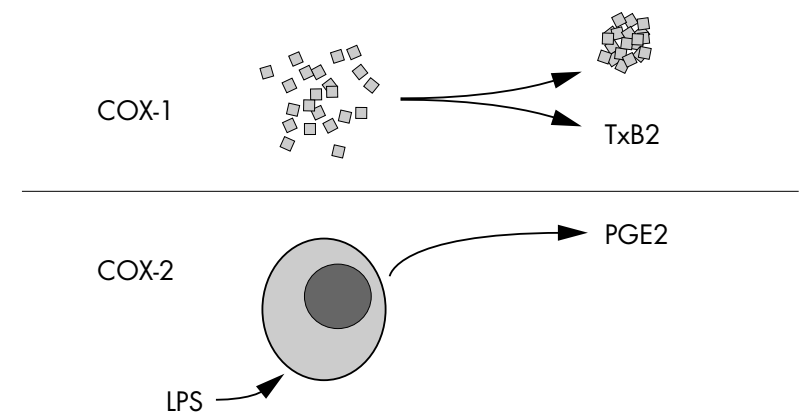

Figure 1 Schematic representation of COX selectivity assay.

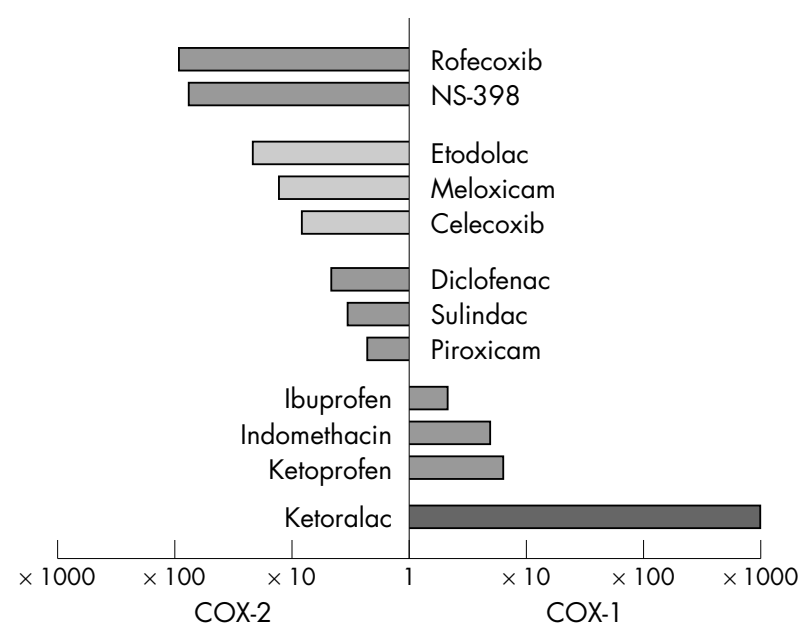

Figure 2 Selectivity ratios of selected drugs. Derived from $I C_{80}$ data. $^{31}$

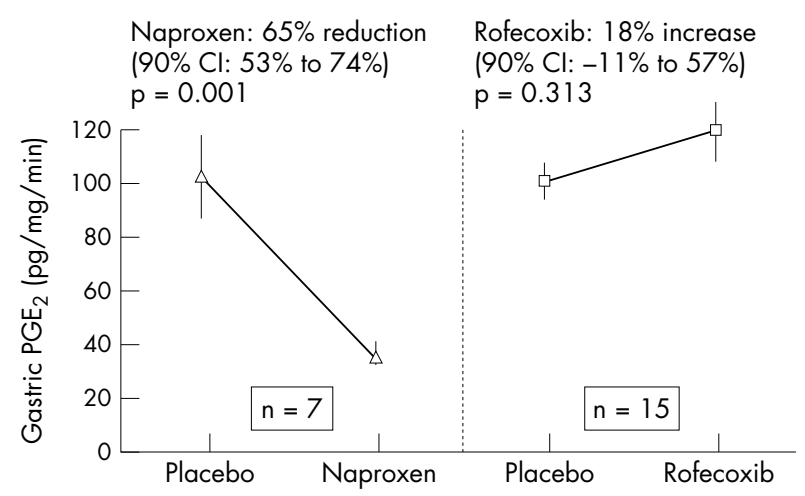

Figure 3 Effect of naproxen $1 \mathrm{~g}$ daily and rofecoxib $50 \mathrm{mg}$ daily on ex vivo gastric mucosal prostaglandin synthesis. Volunteers received placebo, naproxen, or rofecoxib for seven days. Twelve standardised gastric mucosal biopsy samples were taken and prostaglandin synthesis stimulated by vortex mixing. Reproduced from Gastroenterology with permission. ${ }^{33}$

94\% with aspirin $2.6 \mathrm{~g}$ daily ${ }^{35}$ (fig 4). In another study, a lower, though still supratherapeutic dose of rofecoxib $(50 \mathrm{mg})$ had no effect on small intestinal permeability, in contrast to indomethacin $150 \mathrm{mg}$ daily. ${ }^{36}$ Over four weeks, use of rofecoxib $25 \mathrm{mg}$ and $50 \mathrm{mg}$ was associated with no more chronic GI bleeding (measured as excretion of chromium labelled red cells into faeces) than with placebo, in contrast to an enhancement with ibuprofen $2.4 \mathrm{~g}^{37}$

\section{Chronic endoscopic studies in patients}

The effects of rofecoxib 25 and $50 \mathrm{mg}$ daily have been compared with ibuprofen $2.4 \mathrm{~g}$ daily over six months in two

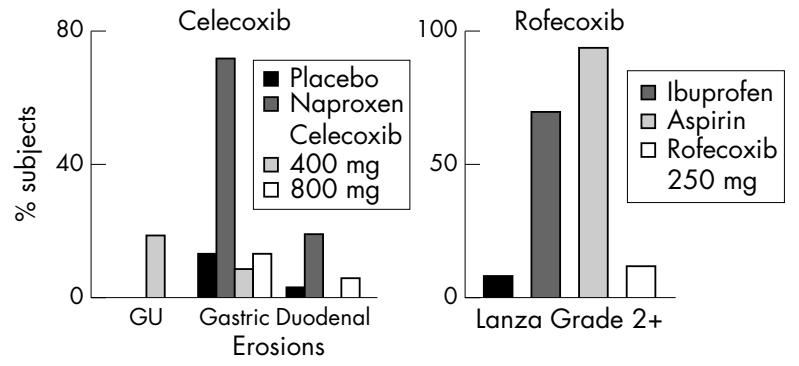

Figure 4 Acute volunteer studies. Lanza grade $2=$ one or more erosions. Data derived from Lanza. ${ }^{354}$

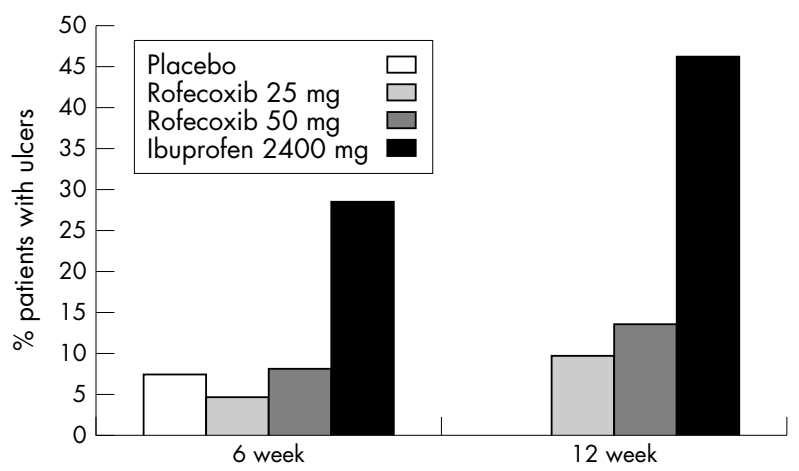

Figure 5 Ulcer development over 12 weeks in patients receiving placebo, rofecoxib $25 \mathrm{mg}$, rofecoxib $50 \mathrm{mg}$, or ibuprofen $2.4 \mathrm{~g}$ daily. Combined data from two studies covering 1516 patients. Reproduced by permission from Arthritis \& Rheumatism. ${ }^{39}$

large studies of osteoarthritis patients. ${ }^{38-41}$ In both studies, a placebo arm was included for the first three months. As both studies were of identical design, they were subjected to a preplanned combined analysis (fig 5). ${ }^{39}$ The rate of ulceration with rofecoxib $25 \mathrm{mg}$ was slightly (though not significantly) lower than with placebo, meeting prespecified criteria for placebo equivalence. The rate of ulceration with rofecoxib $50 \mathrm{mg}$ did not differ significantly from placebo. In contrast, patients taking ibuprofen $2.4 \mathrm{~g}$ daily had a fourfold higher incidence of gastric and duodenal ulcers compared to either placebo or rofecoxib.

\section{"Outcomes" studies of ulcer complications}

Recently, two landmark studies have been published. ${ }^{19} 42$ Both have investigated directly whether use of celecoxib or rofecoxib leads to a reduced incidence of clinically significant ulcers, particularly hospitalisation for ulcer complications, compared to non-selective NSAIDs. In both studies, a higher than therapeutic dose of the COX-2 inhibitor was compared with a normal dose of (and) NSAID comparator(s).

\section{The VIGOR study of rofecoxib}

The VIGOR (VIOXX GI Outcomes Research) study ${ }^{19}$ investigated the incidence of clinically important upper GI (largely ulcer) events with rofecoxib $50 \mathrm{mg}$ compared to naproxen $1 \mathrm{~g}$ daily (table 1). A total of 8076 patients with rheumatoid arthritis were randomised to receive rofecoxib $50 \mathrm{mg}$ daily $(\mathrm{n}=4047)$ or naproxen $500 \mathrm{mg}$ twice daily $(\mathrm{n}=4029)$. Enrolled patients were either over 50 or over 40 and taking chronic steroids (as this is a risk factor). To achieve a broad representative group of patients, there were few exclusion criteria but those using aspirin, anticoagulants, or antiplatelet agents were not allowed in the study. Full dose antiulcer medication was prohibited but over the counter doses of $\mathrm{H}_{2}$ antagonists allowed.

The study aimed to recruit 8000 patients, with a minimum time in the study of six months. Once recruited, patients 
Table 1 VIGOR and CLASS compared

\begin{tabular}{|c|c|c|}
\hline & VIGOR Rofecoxib 50 mg & CLASS Celecoxib 400 mg twice daily \\
\hline Patients & 8076 RA & 8059 OA $(72 \%)+R A$ \\
\hline Aspirin & No & $\leqslant 325 \mathrm{mg}(21 \%)$ \\
\hline NSAIDs & Naproxen $1 \mathrm{~g}$ & $\begin{array}{l}\text { Diclofenac } 150 \mathrm{mg} \\
\text { lbuprofen } 2.4 \mathrm{~g}\end{array}$ \\
\hline Duration & $9.2(13)$ & c.9 (13) - n \\
\hline Primary endpoint & Clinically significant UGI events & Complicated ulcers \\
\hline Secondary endpoint & Complicated events & Clinically significant ulcers \\
\hline Analysis & ITT (life table) & $\begin{array}{l}\text { Crude, censored } \\
\text { ( } 3 \text { days }-6 \text { months) }\end{array}$ \\
\hline
\end{tabular}

stayed in the study until its completion. Patients were not endoscoped at baseline and, to mimic real life, investigators were not given a protocol for the management of GI complaints. Instead, these were investigated according to standard clinical practice at the physician's discretion. In addition there were trial visits at six weeks and four months, and four monthly thereafter until the end of the study and at a 14 day follow up point. Between visits patients received telephone calls.

The primary endpoint was clinical upper GI events (perforation, obstruction, bleeding, or symptomatic ulcer). The secondary endpoint was complicated upper GI events (perforation, ulcer, and major upper GI bleeding). These endpoints were subject to independent blinded assessment by an adjudication committee working to prespecified criteria who classified events as confirmed or unconfirmed. In addition, all episodes of GI bleeding (upper or lower), whether confirmed or unconfirmed, were analysed.

Patients were well matched (table 2). The efficacy of each drug was very similar. Possible upper GI clinical events were reported in 190 patients and confirmed by the adjudication committee in 177. Of these, 53 met the criteria for being confirmed complicated events (43 ulcer complications). Compared to naproxen, rofecoxib use was associated with a reduction in all upper GI events (4.5 to 2.1 per 100 patient years) and also complicated events ( 1.4 to 0.6 per 100 patient years) as well as gastrointestinal bleeding (3.0 to 1.1 per 100 patient years), representing reductions in $54 \%, 57 \%$, and $62 \%$, respectively.

Other risk factors for development of clinical upper GI events were a prior GI event, age $\geqslant 65$ years, and steroid use. Patients using NSAIDs at baseline had reduced risk (presumably because of a process of attrition). The advantage of rofecoxib was maintained in all these subgroups, but was significantly greater in Helicobacter pylori negative than positive individuals. The effect of rofecoxib was significantly greater in $H$ pylori negative than positive individuals. Among individuals without such risk factors, the event rate was reduced by 1.7 per 100 patient years (from 1.9 to 0.2 ) risk reduction $88 \%$ (to $4 \%$ to $96 \%$ ). Among high risk patients the values were 2.6 per 100 patient years (from 5.1 to 2.5 ), a relative risk reduction of $51 \%(35 \%$ to $68 \%)$.

Table 2 Baseline characteristics: VIGOR study

\begin{tabular}{lll}
\hline & $\begin{array}{l}\text { Rofecoxib } \\
(n=4047)\end{array}$ & $\begin{array}{l}\text { Naproxen } \\
(n=4029)\end{array}$ \\
\hline Mean age & $58 \mathrm{yr}$ & $58 \mathrm{yr}$ \\
Female & $80 \%$ & $80 \%$ \\
Prior Gl event & $8 \%$ & $8 \%$ \\
Steroid use & $56 \%$ & $56 \%$ \\
HP seropositive & $42 \%$ & $42 \%$ \\
History of CV disease & $47 \%$ & $46 \%$ \\
\hline
\end{tabular}

The five most common adverse events leading to discontinuation were all abdominal. The incidence of abdominal pain, epigastric discomfort, and any five of the symptoms (dyspepsia, abdominal pain, epigastric discomfort, nausea, or heartburn) were significantly reduced with rofecoxib compared to naproxen. Utilisation of health resources was also significantly reduced with naproxen, with a $50 \%$ reduction in hospitalisation ( 1.3 to 0.6 per 100 patient years), use of GI co-therapy (by 23\%), and of upper GI procedures (by $25 \%$ ).

\section{CELECOXIB}

\section{Selectivity}

A selectivity ratio of 375 has been reported for celecoxib using the recombinant enzyme assay. ${ }^{29}$ By contrast, in the William Harvey modified whole blood assay it only achieved a selectivity ratio of approximately 8 ( fig 2 ), ${ }^{31}$ emphasising the influence of assay system on results. The ability of celecoxib to spare gastric prostaglandin synthesis in humans has not been tested.

\section{Acute gastric injury}

Like rofecoxib, celecoxib has been shown not to cause any more acute gastric injury than placebo ${ }^{43}$ (fig 4 ). The effects of celecoxib on intestinal permeability and chronic blood loss have not been reported.

\section{Chronic endoscopic studies in patients}

The effects of celecoxib in a daily dose range of 100 to $800 \mathrm{mg}$ daily have been compared with diclofenac ( $150 \mathrm{mg}$ daily) and naproxen ( $1 \mathrm{~g}$ daily) over periods of up to three months in six studies in both rheumatoid and osteoarthritis patients (fig 6). ${ }^{40}{ }^{414}$ These studies have varied in design, some conducting sequential endoscopy, others dependent on a single endoscopy at the end of the trial. Consequently absolute rates of ulceration have differed. Nevertheless, in five studies, celecoxib caused significantly less mucosal injury than the active NSAID and not significantly more than placebo. In a sixth study, the rate of ulceration with celecoxib did not differ significantly from a (lower than expected) ulcer rate with diclofenac.

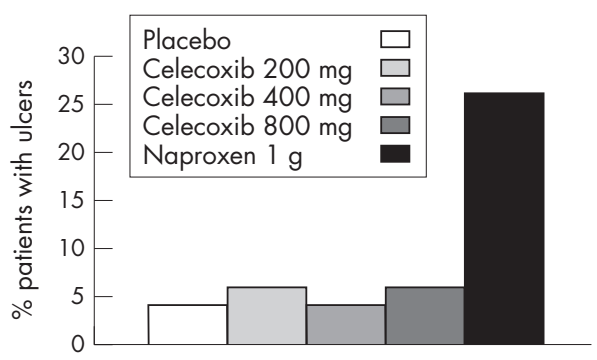

Figure 6 Ulcer development in patients receiving celecoxib in endoscopic studies. Reproduced with kind permission of the Journal of the American Medical Association. ${ }^{44}$ 


\section{The CLASS study of celecoxib}

In the Celecoxib Long-term Arthritis Safety Study (CLASS), ${ }^{45}$ celecoxib $400 \mathrm{mg}$ twice daily was compared with ibuprofen $800 \mathrm{mg}$ three times daily or diclofenac $700 \mathrm{mg}$ twice daily. The size and design of this study was very similar to the VIGOR study (table 1) but there were some important differences. In particular, aspirin use for cardiovascular prophylaxis $(\leqslant 325$ mg daily) was permitted and was used by $21 \%$ of patients. Patients with both osteoarthritis (72\%) and rheumatoid arthritis $(28 \%)$ were eligible for entry into the study. Although the study lasted for a median of nine months and a maximum of 13 months, only data censored for the first six months of treatment were reported. As with VIGOR, both ulcer complications and symptomatic ulcers were endpoints, but complications were the primary endpoint and all ulcers the secondary endpoint.

A total of 8059 patients were randomised, and 7968 received at least one dose of study drug (3987 celecoxib, 1985 ibuprofen, 1996 diclofenac). There was a higher drop out rate than in the VIGOR study, with only 4573 patients receiving treatment for six months (57\%), and probably as a result, fewer endpoints and lower power.

The overall pattern of results in the CLASS study, as reported in the Journal of the American Medical Association, was similar to that seen in VIGOR. However, the reduction in upper GI ulcer complications ( $1.5 \%$ to $0.76 \%$ ) did not reach statistical significance $(p=0.09)$. This in part reflected the smaller number of events and in part the influence of aspirin in one fifth of the patients (see below). The reduction in all ulcers $(3.54 \%$ to $2.08 \%)$ was statistically significant $(\mathrm{p}=0.02)$. These data relate to the first six months of the study. Full data revealed at the FDA hearings (7 February 2001), however, showed no difference in the rate of ulcer complications on celecoxib compared to ibuprofen and diclofenac combined over the full 13 months of the study $(p=0.45)$.

\section{Other effects}

The overall incidence of GI symptoms was significantly lower in patients taking celecoxib than in those taking diclofenac/ ibuprofen. Fewer patients on celecoxib became anaemic than on ibuprofen/diclofenac, whether or not they were also taking aspirin. Liver function test abnormalities were more common with diclofenac than the other drug. The overall incidence of renal adverse effects was significantly lower in patients receiving celecoxib than ibuprofen/diclofenac.

\section{GI OR CARDIOVASCULAR EVENTS-TRADE OFFS BETWEEN THE DEVIL AND THE DEEP BLUE SEA?}

Attention has focused on differences in cardiovascular outcomes in the VIGOR ${ }^{19}$ and CLASS ${ }^{45}$ studies. In the CLASS study, the GI advantages of celecoxib over ibuprofen/ diclofenac were lost in patients that were also taking aspirin. ${ }^{45}$ However, the numbers involved are too small to say whether this indicated no benefit or a reduced benefit in such patients. In the VIGOR study, patients did not use aspirin. ${ }^{19}$ In patients who had indications for aspirin use but who were enrolled in the trial, there was an increased incidence of myocardial infarction compared to naproxen. Whether this indicates that COX-2 inhibitors promote myocardial infarction or that (some) NSAIDs have an aspirin like effect in preventing it, is currently uncertain. However, it is worth noting that the overall proportion of patients on celecoxib, rofecoxib, diclofenac, and ibuprofen in the two trials who experienced myocardial infarction was similar. While big differences in trial population mean that conclusions must be cautious, it is naproxen that appears to differ from the other drugs, with a lower myocardial infarction rate. Several functional studies suggest that naproxen has a sufficiently profound effect on serum thromboxane and platelet function over 24 hours to exert an aspirin like effect on cardiovascular pathology. ${ }^{33} 46$

\section{RENAL ADVERSE EVENTS-TRUE DEEP BLUE SEA}

The adverse effects of NSAIDs on salt and water retention ${ }^{18}$ and the consequent increase in heart failure, ${ }^{47} 48$ have received less attention than their ability to damage the GI tract, but may be at least as important. Both COX-1 and COX-2 are expressed in the kidney, to a different extent at different sites. ${ }^{139}$ COX-2 is expressed constitutively and plays a central role in renin release ${ }^{50-53}$ and sodium handling. ${ }^{49}$ Although COX-1 is also expressed in the kidney, as yet clear differences between non-selective NSAIDs and COX-2 inhibitors which would indicate a critical role for COX-1 have yet to emerge. Consequently, COX-2 inhibitors should be regarded as retaining all of the toxicities of NSAIDs with regard to kidney function. Indeed, because dosing may be less constrained by gastrointestinal considerations, COX-2 inhibitors have the potential in practice to cause more renal related pathology than non-selective NSAIDs. In theory and in practice such pathology is dose related.

\section{CELECOXIB VERSUS ROFECOXIB}

One might expect differences between individual drugs, related to the effective potency of marketed doses, to emerge, both for efficacy and safety. Moreover, because celecoxib is very poorly soluble in aqueous medium, its absorption is not dose related, and proportional bioavailability reduces as dose increases, in contrast to rofecoxib. Several head to head comparisons illustrate these relations. In one very small study, celecoxib $200 \mathrm{mg}$ daily was shown to be of similar efficacy to rofecoxib $25 \mathrm{mg}$ daily, though a type 2 error was possible. In another larger study, rofecoxib $25 \mathrm{mg}$ was superior to both paracetamol $4 \mathrm{~g}$ daily and celecoxib $200 \mathrm{mg}$ daily in an overall assessment of osteoarthritis. Rofecoxib $12.5 \mathrm{mg}$ daily was superior to paracetamol and there was a non-significant advantage to this dose of rofecoxib compared to celecoxib 200 mg daily. ${ }^{54}$

Overall, therefore, rofecoxib $25 \mathrm{mg}$ is probably a higher effective dose than celecoxib $200 \mathrm{mg}$, as a result of selected dose and bioavailability considerations. It is, therefore, not surprising that, when given to treated hypertensive patients, rofecoxib $25 \mathrm{mg}$ resulted in a higher blood pressure than celecoxib $200 \mathrm{mg}$ daily. ${ }^{55}$ The elevation on rofecoxib was approximately $3 \mathrm{~mm}$, within the $5 \mathrm{~mm}$ range quoted for nonselective NSAIDs. ${ }^{18}$ In some countries such as the UK, many patients take quite low doses of NSAIDs, particularly ibuprofen $\leqslant 1200 \mathrm{mg}$ daily. As it is probable that available doses of COX-2 inhibitors may represent higher effective levels of COX-2 inhibition than such low doses of ibuprofen, there may be particular problems regarding fluid retention in patients switched from such dosing regimens.

There have been claims that fluid retention is not a property of all COX-2 inhibitors, but the data do not support this proposition and it seems unlikely that one COX-2 inhibitor would escape mechanism based toxicities while the other would have idiosyncratic effects similar to those mechanism based impacts.

\section{COX-2 SELECTIVITY OF OTHER DRUGS}

Of the older NSAIDs, only diclofenac has activity that is marginally COX-2 selective. ${ }^{31}$ This does not appear to be sufficient to give it a distinct safety advantage (although in one study ulceration on diclofenac $150 \mathrm{mg}$ daily was not significantly different from celecoxib ${ }^{41}$ ). Although not designed to be COX-2 selective, three relatively recently introduced NSAIDs, meloxicam, etodalac, and nimesulide, have serendipitously been found to be COX-2 selective.

\section{Meloxicam}

Meloxicam's selectivity has been the subject of some debate. However, in a large comparison in the William Harvey modified whole blood assay, it achieved a selectivity ratio 
similar to celecoxib. ${ }^{31}$ Modelling and direct in vivo studies have suggested that meloxicam remains selective when given at 7.5 $\mathrm{mg}$ daily but is less selective at $15 \mathrm{mg}$ daily. ${ }^{56} 57$ In one study, meloxicam caused no change in gastric mucosal prostaglandin synthesis, but neither did piroxicam, ${ }^{58}$ raising questions about the validity of the method.

Acute gastric injury

In volunteers treated for four weeks, meloxicam $15 \mathrm{mg}$, like piroxicam $20 \mathrm{mg}$ caused significant mucosal damage, whereas the change from baseline with meloxicam $7.5 \mathrm{mg}$ was not significant. ${ }^{59}$ While studies have been small, injury levels with the lower dose of meloxicam, $7.5 \mathrm{mg}$, have been very low.

Endoscopy studies in patients

Meloxicam has not been subject to the same level of prospective large scale systematic endoscopic evaluation in long term patient trials, as have rofecoxib and celecoxib.

\section{Patient studies of tolerability}

Meloxicam has been compared with NSAIDs in eight patient studies, none of them systematically controlled by endoscopy. In six relatively small studies meloxicam did not differ from the NSAID comparator. ${ }^{60}$ In two large studies, each enrolling over 8000 patients, meloxicam $7.5 \mathrm{mg}$ was given for one month and compared to diclofenac or piroxicam. ${ }^{61}{ }^{62}$ In both of these studies meloxicam was significantly better tolerated than the comparator. This was particularly evident for gastrointestinal adverse effects. Whether this is attributable to COX-2 selectivity, relative differences in dose or some other effect is not clear. In three studies comparing meloxicam with placebo, there was no significant difference in the rate of gastrointestinal adverse events.

\section{Gl outcomes on meloxicam}

There have been no prospective, systematic studies of outcomes on meloxicam. An ad hoc analysis of two large trials suggested possible reductions, but should be treated with caution as the number of events were small and the analyses were not prespecified. Two epidemiological studies have been published. One found a lower rate of ulcer complications than for comparator NSAIDs, while the other found no difference. ${ }^{63}{ }^{64}$

\section{Etodalac}

Selectivity

In the William Harvey modified assay, etodalac was of similar selectivity to meloxicam and celecoxib (fig 2). In two studies, etodalac was shown not to inhibit human gastric mucosal prostaglandin synthesis, in contrast to naproxen. ${ }^{65} 66$

\section{Acute endoscopic studies}

Therapeutic doses of etodalac have been shown to cause lower levels of mucosal injury than naproxen $1 \mathrm{~g}$ daily. Supratherapeutic doses of etodalac have not been tested.

\section{Patient studies}

At doses of 100 to $1000 \mathrm{mg}$ per day, etodalac has shown a reduction in overall and GI adverse events compared to NSAID comparators such as aspirin, naproxen, and diclofenac. ${ }^{65}$ 67-72

\section{OVERALL EVALUATION}

While there are data favouring the notion that all five compounds that are selective for $\mathrm{COX}-2$ cause less gastric damage than non-selective NSAIDs, the evidence is much greater and more systematic for celecoxib and rofecoxib. The number of patients involved in the prospective evaluation of these two drugs is sufficient to allow a confident confirmation of the COX-2 hypothesis, that selectivity would be associated with little or no gastroduodenal injury. In addition, prospective outcome studies have shown that this benefit translates into real clinical benefits. Why the reductions in GI outcomes with these drugs is rather less than reductions in endoscopic injury is not clear, but is at least as likely to reflect design issues as residual drug toxicity. Nevertheless, it is clear that use of COX-2 inhibitors is associated with a residual level of ulceration and ulcer complications, that is attributable to well identifiable risk factors. To some extent this observation undermines the truism that has developed, that COX-2 inhibitors should be used in high risk patients. In fact, it is in low risk patients that these drugs could offer the most benefit. The absolute reduction in the risk of endoscopic ulceration and in ulcer complications in these patients is not far short of that seen in high risk patients ( 1.7 versus 2.5 events per 100 patient years), although confidence intervals are wide. Moreover, the residual risk that is attributable to other factors is very low, allowing the prescriber considerable confidence that a low risk patient taking a COX-2 inhibitor is extremely unlikely to experience ulcer related disease. By contrast, risk is only halved in high risk patients. Attention is still needed to deal with residual risk factors such as $H$ pylori, past history, steroid use, old age, or aspirin. These patients may therefore need additional treatment, such as prophylaxis with a proton pump inhibitor, that potentially renders the switch to a COX-2 inhibitor superfluous.

\section{REFERENCES}

1 Tramer MR, Moore RA, Reynolds DJM, McQuay HJ. Quantitative estimation of rare adverse events which follow a biological progression: a new model applied to chronic NSAID use. Pain 2000;85:169-82.

2 Hawkey CJ, Hudson N. Mucosal injury caused by drugs, chemicals and stress. In: Haubrich WS, Schaffner F, Berk JE, eds. Bockus gastroenterology, Vol. 2, 5th edn. Philadelphia: WB Saunders, 1994:656-99.

3 Gabriel SE, Jaakkimainen L, Bombardier C. Risk for serious gastrointestinal complications related to use of nonsteroidal anti-inflammatory drugs: a meta-analysis. Ann Intern Med 1991;115:787-96.

4 Garcia Rodriguez LA, Jick $\mathrm{H}$. Risk of upper gastrointestinal bleeding and perforation associated with individual non-steroidal anti-inflammatory drugs. Lancet 1994;343:769-72.

5 Henry D, Lim L-Y, Garcia Rodriguez LA, et al. Variability in risk of gastrointestinal complications with individual non-steroidal anti-inflammatory drugs: results of a collaborative meta-analysis. BM 1996;312:1563-6.

6 Langman MJ, Weil J, Wainwright $P$, et al. Risks of bleeding peptic ulcer associated with individual non-steroidal anti-inflammatory drugs. Lancet 1994;343: 1075-8.

7 Piper JM, Ray WA, Daugherty JR, et al. Corticosteroid use and peptic ulcer disease: role of nonsteroidal anti-inflammatory drugs. Ann Intern Med 1991;114:735-40.

8 Shorr RI, Ray WA, Daugherty JR, et al. Concurrent use of nonsteroidal anti-inflammatory drugs and oral anticoagulants places elderly persons at high risk for hemorrhagic peptic ulcer disease. Arch Intern Med 1993; 153: 1665-70.

9 Weil J, Langman M, Wainwright $P$, et al. Peptic ulcer bleeding: accessory risk factors and interactions with non-steroidal anti-inflammatory drugs. Gut 2000;46:27-31.

10 Bjorkman D. Nonsteroidal anti-inflammatory drug-associated toxicity of the liver, lower gastrointestinal tract, and esophagus. Am J Med 1998; 105:17S-21S.

11 Schoenfeld P, Kimmey MB, Scheiman J, et al. Review article: nonsteroidal anti-inflammatory drug-associated gastrointestinal complications-guidelines for prevention and treatment. Aliment Pharmacol Ther 1999;13:1273-85.

12 Gurwitz JH, Avorn J, Bohn RL, et al. Initiation of antihypertensive treatment during nonsteroidal anti-inflammatory drug therapy. JAMA 1994;272:781-6.

13 Brater DC. Effects of nonsteroidal anti-inflammatory drugs on renal function: focus on cyclooxygenase-2-selective inhibition. Am J Med 1999; 107:70S-71S.

14 Nantel F, Meadows E, Denis D, et al. Immunolocalization of cyclooxygenase-2 in the macula densa of human elderly. FEBS Lett 1999;457:475-7.

15 Yang T, Singh I, Pham H, et al. Regulation of cyclooxygenase expression in the kidney by dietary salt intake. Am J Physiol 1998;274:F481-9.

16 Schnermann J. Juxtaglomerular cell complex in the regulation of renal salt excretion. Am J Physiol 1998;274:R263-79.

17 Yang T, Schnermann JB, Briggs JP. Regulation of cyclooxygenase-2 expression in renal medulla by tonicity in vivo and in vitro. Am J Physiol 1999;277:F1-9.

18 Johnson AG, Nguyen TV, Day RO. Do nonsteroidal anti-inflammatory drugs affect blood pressure? A meta-analysis. Ann Intern Med 1994; 121:289-300. 
19 Bombardier C, Laine L, Reicin A, et al. Comparison of uppe gastrointestinal toxicity of rofecoxib and naproxen in patients with rheumatoid arthritis. N Engl J Med 2000;343:1520-8.

20 GPRD. Data presented to FDA hearings. 8 February 2001.

21 Vane JR. Inhibition of prostaglandin synthesis as a mechanism of action for aspirin-like drugs. Nat New Biol 1971;231:232-5.

22 Hawkey CJ. Nonsteroidal anti-inflammatory drug gastropathy. Gastroenterology 2000;1 19:521-35.

23 Simmons DL, Levy DB, Yannoni Y, Erikson RL. Identification of a phorbol ester-repressible v-src-inducible gene. Proc Natl Acad Sci U S A 1989;86:1178-82

24 Xie W, Chipman JG, Robertson DL, et al. Expression of a mitogen-responsive gene encoding prostaglandin synthase is regulated by mRNA splicing. Proc Natl Acad Sci U S A 1991:88:2692-6.

25 Fu JY, Masferrer JL, Seibert K, et al. The induction and suppression of prostaglandin $\mathrm{H} 2$ synthase (cyclooxygenase) in human monocytes. J Biol Chem 1990;265: 16737-40.

26 Wallace JL, McKnight W, Reuter BK, et al. NSAID-induced gastric damage in rats: requirement for inhibition of both cyclooxygenase 1 and 2. Gastroenterology 2000;1 19:706-14.

27 Willoughby DA, Moore AR, Colville-Nash PR. COX-1, COX-2, and COX-3 and the future treatment of chronic inflammatory disease. Lancet 2000;355:646-8.

28 Simmons DL, Botting RM, Robertson PM, et al. Induction of an acetaminophen-sensitive cyclooxygenase with reduced sensitivity to nonsteroid antiinflammatory drugs. Proc Natl Acad Sci U S A 1999;96:3275-80.

29 Hawkey CJ. COX-2 inhibitors. Lancet 1999;353:307-14.

30 Brooks P, Emery P, Evans JF, et al. The International Consensus Meeting on the Mode of Action of COX-2 Inhibition, 5-6 December 1997. Interpreting the clinical significance of the differential inhibition of cyclooxygenase-1 and cyclooxygenase-2. Rheumatology 1999;38:779-88.

31 Warner TD, Giuliano F, Vojnovic I, et al. Nonsteroid drug selectivities for cyclo-oxygenase-1 rather than cyclo-oxygenase-2 are associated with human gastrointestinal toxicity: a full in vitro analysis [published erratum appears in Proc Natl Acad Sci U S A 1999;96:9666]. Proc Natl Acad Sci U S A 1999;96:7563-8

32 Patrignani $\mathbf{P}$, Panara MR, Greco A, et al. Biochemical and pharmacological characterization of the cyclooxygenase activity of human blood prostaglandin endoperoxide synthases. J Pharmacol Exp Ther 1994;271:1705-12

33 Wight N, Gottesdiener K, Garlick NM, et al. Rofecoxib, a COX-2 inhibitor, does not inhibit human gastric mucosal prostaglandin production. Gastroenterology 2001;120:867-73.

34 Cryer B, Gottesdiener K, Gertz BJ, et al. In vivo effects of rofecoxib, a new cyclooxygenase (COX)-2 inhibitor, on gastric mucosal prostaglandin (PG) and serum thromboxane B2 (TBX2) synthesis in healthy humans. Gastroenterology 1999;116:A141.

35 Lanza FL, Rack MF, Simon TJ, et al. Specific inhibition of cyclooxygenase-2 with MK-0966 is associated with less gastroduodenal damage than either aspirin of ibuprofen. Aliment Pharmacol Ther 1999;13:761-7

36 Sigthorsson G, Crane R, Simon T, et al. COX-2 inhibition with rofecoxib does not increase intestinal permeability in healthy subjects: a double blind crossover study comparing rofecoxib with placebo and indomethacin. Gut 2000;47:527-32

37 Hunt RH, Bowen B, Mortensen ER, et al. A randomized trial measuring fecal blood loss after treatment with rofecoxib, ibuprofen, or placebo in healthy subjects. Am J Med 2000;109:201-6.

38 Laine L, Harper S, Simon T, et al. A randomized trial comparing the effect of rofecoxib, a cyclooxygenase 2-specific inhibitor, with that of ibuprofen on the gastroduodenal mucosa of patients with osteoarthritis. Gastroenterology 1999;117:776-83.

39 Hawkey C, Laine L, Simon T, et al. Comparison of the effect of rofecoxib (a cyclooxygenase 2 inhibitor), ibuprofen, and placebo on the gastroduodenal mucosa of patients with osteoarthritis: a randomized, double-blind, placebo-controlled trial. Arthritis Rheum 2000;43:370-7.

40 FDA. Rofecoxib. Website: http://www.fda.gov/ohrms/dockets/ac/01/ slides/3677s2_01_sponsor.pdf. 2001.

41 FDA. Celecoxib Website: http://www.fda.gov/ohrms/dockets/ac/01/ slides/3677s1_01_sponsor.pdf. 2001

42 Silverstein FE, Faich G, Goldstein JL, et al. Gastrointestinal toxicity with celecoxib vs nonsteroidal anti-inflammatory drugs for osteoarthritis and rheumatoid arthritis: the CLASS study: a randomized controlled trial. Celecoxib long-term arthritis safety study. JAMA 2000;284:1247-55.

43 Simon LS, Lanza FL, Lipsky PE, et al. Preliminary study of the safety and efficacy of SC-58635, a novel cyclooxygenase 2 inhibitor. Efficacy and safety in two placebo-controlled trials in osteoarthritis and rheumatoid arthritis, and studies of gastrointestinal and platelet effects. Arthritis Rheum 1998;41:1591-602.

44 Simon LS, Weaver AL, Graham DY, et al. Anti-inflammatory and upper gastrointestinal effects of celecoxib in rheumatoid arthritis: a randomized controlled trial. JAMA 1999:282:1921-8.

45 Silverstein FE, Faich G, Goldstein JL, et al. Gastrointestinal toxicity with celecoxib vs nonsteroidal anti-inflammatory drugs for osteoarthritis and reumatoid arthritis: the CLASS study: a randomized controlled trial. JAMA 2000;284: 1247-55
46 Merck. Data presented to FDA Ft.

47 Henry D, Page J, Whyte I, et al. Consumption of non-steroidal anti-inflammatory drugs and the development of functional renal impairment in elderly subjects. Results of a case-control study. Br J Clin Pharmacol 1997:44:85-90.

48 Page J, Henry D. Consumption of NSAIDs and the development of congestive heart failure in elderly patients: an underrecognized public health problem. Arch Intern Med 2000:160:777-84.

49 Swan SK, Rudy DW, Lasseter KC, et al. Effect of cyclooxygenase-2 inhibition on renal function in elderly persons receiving a low-salt diet. A randomized, controlled trial. Ann Intern Med 2000;133:1-9.

50 Harris RC, McKanna JA, Akai Y, et al. Cyclooxygenase-2 is associated with the macula densa of rat kidney and increases with salt restriction. J Clin Invest 1994;94:2504-10.

51 Wolf K, Castrop H, Hartner A, et al. Inhibition of the renin-angiotensin system upregulates cyclooxygenase-2 expression in the macula densa. Hypertension 1999;34:503-7.

52 Harris RC. Macula densa signalling-a potential role of cyclooxygenase-2 (COX-2)? Nephrol Dial Transplant 2000;15:1504-6.

53 McGiff JC, Quilley J. 20-HETE and the kidney: resolution of old problems and new beginnings. Am J Physiol 1999:277:R607-23.

54 Geba GP, Weaver AL, Schnitzer TJ, et al. A clinical trial comparing rofecoxib to celecoxib and acetaminophen in the treatment of osteoarthritis (OA): early efficacy results. Ann Rheum Dis 2000;59:A133.

55 Whelton A, Fort JG, Puma JA et al. Cyclooxygenase-1 specific inhibitors and cardiorenal function: a randomized, controlled trial of celecoxib and rofecoxib in older hypertensive osteoarthritis patients. Am J Ther 2001;8:85-95

56 Patrignani $\mathbf{P}$, Panara MR, Sciulli MG, et al. Differential inhibition of human prostaglandin endoperoxide synthase- 1 and -2 by nonsteroidal anti-inflammatory drugs. J Physiol Pharmacol 1997;48:623-31

57 Panara MR, Renda G, Sciulli MG, et al. Dose-dependent inhibition of platelet cyclooxygenase-1 and monocyte cyclooxygenase-2 by meloxicam in healthy subjects. J Pharmacol Exp Ther 1999;290:276-80.

58 Lipscomb GR, Wallis N, Armstrong G, et al. Gastrointestinal tolerability of meloxicam and piroxicam: a double-blind placebo-controlled study. $\mathrm{Br}$ J Clin Pharmacol 1998;46:133-7.

59 Patoia L, Santucci L, Furno P, et al. A 4-week, double-blind, parallel-group study to compare the gastrointestinal effects of meloxicam $7.5 \mathrm{mg}$, meloxicam $15 \mathrm{mg}$, piroxicam $20 \mathrm{mg}$ and placebo by means of faecal blood loss, endoscopy and symptom evaluation in healthy volunteers. Br J Rheumatol 1996;35:61-7.

60 Distel M, Mueller C, Bluhmki E, et al. Safety of meloxicam: a global analysis of clinical trials. BrJ Rheumatol 1996;35:68-77.

61 Hawkey C, Kahan A, Steinbruck K, et al. Gastrointestinal tolerability of meloxicam compared to diclofenac in osteoarthritis patients. Internationa MELISSA study group. Meloxicam large-scale international study safety assessment. BrJ Rheumatol 1998;37:937-45.

62 Dequeker J, Hawkey C, Kahan A, et al. Improvement in gastrointestinal tolerability of the selective cyclooxygenase (COX)-2 inhibitor, meloxicam compared with piroxicam: results of the safety and efficacy large-scale evaluation of COX-inhibiting therapies (SELECT) trial in osteoarthritis. $\mathrm{Br} J$ Rheumatol 1998;37:946-51.

63 Degner F, Sigmund R, Zeidler H. Efficacy and tolerability of meloxicam in an observational, controlled cohort study in patients with rheumatic disease. Clin Ther 2000;22:400-10.

64 Jick SS. The risk of gastrointestinal bleed, myocardial infarction, and newly diagnosed hypertension in users of meloxicam, diclofenac, naproxen, and piroxicam. Pharmacotherapy 2000;20:741-4.

65 Taha AS, Mclaughlin S, Holland PJ, et al. Effect on gastric and duodenal mucosal prostaglandins of repeated intake of therapeutic doses of naproxen and etodolac in rheumatoid arthritis. Ann Rheum Dis 1990;49:354-8.

66 Laine L, Cominelli F, Sloane R, et al. Interaction of NSAIDs and Helicobacter pylori on gastrointestinal injury and prostaglandin production: a controlled double-blind trial. Aliment Pharmacol Ther 1995; $9: 127-35$

67 Jacob G. Long-term evaluation of the efficacy and safety of etodolac in the treatment of rheumatoid arthritis. Adv Ther 1985;2:82-95.

68 Ciompi ML, Puccetti L, Bazzichi L, et al. Etodolac versus diclofenac: double-blind cross-over study in rheumatoid arthritis. Int J Clin Pharmacol Res 1989;9:217-22.

69 Porzio F. Double-blind comparison of etodolac sustained-release tablets and diclofenac sustained-release tablets in patients with rheumatoid arthritis. Curr Ther Res Clin Exp 1993;53:144-53.

70 Lightfoot R. Comparison of the efficacy and safety of etodolac and piroxicam in patients with rheumatoid arthritis. Etodolac Study 326, Rheumatoid Arthritis Investigators Group. J Rheumatol Suppl 1997;47:10-16.

71 Perpignano G, Bogliolo A, Puccetti L. Double-blind comparison of the efficacy and safety of etodolac SR $600 \mathrm{mg}$ uid and of tenoxicam $20 \mathrm{mg}$ vid in elderly patients wtih osteoarthritis of the hip and of the knee. Int J Clin Pharmacol Res 1994;14:203-16.

72 Rogind $\mathbf{H}$. Comparison of etodolac and piroxicam in patients with osteoarthritis of the hip or knee: a prospective, randomised, double-blind, controlled multicentre study. Clin Drug Invest 1997;13:66-75. 